\title{
Comparison of Preoperative and Postoperative Parecoxib Administration for Pain Control Following Major Spine Surgery
}

\author{
Rayakorn Moonla ${ }^{1}$, Adisorn Threetipayarak ${ }^{2}$, Chininthon Panpaisarn ${ }^{1}$, Nida Pattayaruk ${ }^{3}$, \\ Unchana Kaewkam ${ }^{3}$, Nipawan Jumpalee ${ }^{3}$, Jatupong Panwilai ${ }^{3}$ \\ ${ }^{1}$ Department of Anesthesiology, Chiangmai Neurological Hospital, Chiangmai, Thailand \\ ${ }^{2}$ Department of Pharmacy, Chiangmai Neurological Hospital, Chiangmai, Thailand \\ ${ }^{3}$ Department of Nurse Anesthesia, Chiangmai Neurological Hospital, Chiangmai, Thailand
}

Study Design: Prospective randomized, double-blind controlled trial.

Purpose: Here, we aim to compare the efficacy and safety of pain control between pre- and postoperative parecoxib administration in patients who have undergone major spine surgery.

Overview of Literature: Several studies have compared the efficacy of pre- and postoperative administration of parecoxib, which led to inconclusive results owing to variation in operative time. Preincisional parecoxib administration reduces inflammatory response in major spine surgery requiring longer operative time; however, it may not reduce pain as much as parecoxib administration immediately after surgery would.

Methods: Totally, 127 patients who underwent major spine surgery were randomly divided into three groups: pre-group, which received $40 \mathrm{mg}$ parecoxib before skin incision and at 12 and 24 hours after the first dose; post-group, which received the same dose at wound closure and at 12 and 24 hours after the first dose; and control group, which did not receive any parecoxib. Efficacy and safety of parecoxib were measured based on pain score, morphine consumption, and side effects from both morphine and parecoxib at 24 hours after surgery.

Results: Initial postoperative pain score, postoperative pain score at rest, and accumulative morphine consumption at 24 hours after surgery were similar between the pre- and post-groups. Despite the significantly lower pain score and morphine consumption in both pre- and post-groups compared with the control group, cumulative morphine consumption at 24 hours after surgery was reduced by approximately $50 \%$ in the pre-group and $46 \%$ in the post-group compared. Analgesic-related complication incidence was similar in all groups.

Conclusions: The timing of parecoxib administration, either before or after major spinal surgery, did not affect the safety and analgesic efficacy of pain management.

Keywords: Spine; Orthopedics; Postoperative pain; Pain management; Non-steroidal anti-inflammatory agents

Received Aug 31, 2017; Revised Nov 30, 2017; Accepted Feb 18, 2018

Corresponding author: Rayakorn Moonla

Department of Anesthesiology, Chiangmai Neurological Hospital, 2 Suthep road, Suthep, Meangchiangmai, Chiangmai, 50200 Thailand Tel: +66-053-920-200, Fax: +66-053-320-202, E-mail: raya-korn@hotmail.com 


\section{Introduction}

The frequency of spinal surgery has increased during the last decade, particularly in patients aged $\geq 65$ years $[1,2]$, who are prone to having multiple underlying diseases and degenerative vital organs. Performing any spinal operation in such patients could involve multiple spinal levels, thus requiring multilevel instrumentation and prolonged operative time leading to more extensive tissue injury, which is often associated with severe postoperative pain and consumption of large amounts of opioids. Opioids have well-known side effects, such as respiratory distress, sedation, nausea, vomiting, debility, and slower postoperative rehabilitation [3], which can interfere with the administration of appropriate doses of pain relief medication and, in turn, can result in the development of chronic pain [4].

Adequate postoperative pain control reduces postsurgical complications and subsequent chronic pain. This has been studied using various methods $[5,6]$. Postoperative pain control aims to relieve the pain with fewer analgesicassociated complications, where the proper cost-effectiveness is under concern. The initiation of pain management before surgery is known as preventive analgesia. This can be performed by providing medication, a procedure, or any intervention to patients prior to the procedure [7-9]. The simplest method involves using analgesic medicine, such as nonsteroidal anti-inflammatory drugs (NSAIDs). Parecoxib is a cyclooxygenase-2 selective inhibitor, which reduces central sensitization and inflammation of peripheral nociceptors [10]. It helps decrease opioid usage, with low risk of peptic ulceration. Furthermore, parecoxib does not affect platelet function and therefore does not cause increased bleeding during or after surgery. The recommended dose of parecoxib for acute postoperative pain is $40 \mathrm{mg}$ intravenously or intramuscularly, followed by 20 or $40 \mathrm{mg}$ every 6-12 hours, up to maximum $80 \mathrm{mg} /$ day. Perceptible analgesic effect onset occurred within 7-13 minutes, clinically meaningful analgesia within 23-39 minutes, and a peak effect within 2 hours. The duration of analgesia depends on the dose and clinical pain model and ranges from 6 to $>12$ hours. Parecoxib has a plasma half-life of approximately 22 minutes and is rapidly and almost completely converted [11]. Moreover, it is available for parenteral administration and offers advantages over oral administration when patients are unable to swallow, such as during the operative or immediate postoperative period.
In previous studies concerning the efficacy of analgesia, administration of parecoxib was compared between preand postoperative periods [12-15]. These results were inconclusive owing to the interference between the duration of analgesia (6-12 hours) and the uncertain (too short or too long) operation time. If parecoxib is administered prior to short surgical procedures, patients should have complete analgesia when they recover from the anesthesia after surgery. However, if the operative time is longer than the duration of analgesia, the group of patients wherein parecoxib is administered prior to surgery may experience more pain than the group with a shorter operation time after recovering from anesthesia.

Here, we aimed to compare the efficacy and safety of postoperative pain control between pre- and postoperative parecoxib administration in patients who have undergone major spine surgery; this can take as short as 2.5 hours to as long as 6 or 7 hours, occasionally even longer. This study was designed to test the hypothesis that parecoxib administered immediately after surgery would improve postoperative analgesia better than when administered before surgery (as the peak analgesic effect of parecoxib is approximately 2 hours). Particularly, when the surgical time is $>6$ hours, parecoxib blood level may be lower than the effective analgesic level.

\section{Materials and Methods}

This study was a double-blind, three-group, prospective, randomized controlled study conducted at a hospital between February 2016 and May 2017. The sample size $(n=26)$ was calculated using hypothesis testing of two independent means [13]. Written informed consent was obtained from all participants prior to the experiment under EC 005-59 and EC 003-60 protocols approved by Chiangmai Neurological Hospital Human Research Ethics Committee; the study was conducted in accordance with ethical principles of the Declaration of Helsinki.

Data were collected from 144 patients who underwent major spinal surgery. Briefly, the inclusion criteria were as follows: (1) age, 21-88 years; (2) male or female sex; (3) not pregnant or lactating; (4) fully conscious and able to agree with the study protocol; (5) major spinal surgery (expected to be a long surgery) involving $>2$ spinal levels or using spinal fixation devices; (6) attended by a neurosurgeon with $>3$ years' experience in spinal surgery; and (7) ASA (American Society for Anesthesiologists) physi- 


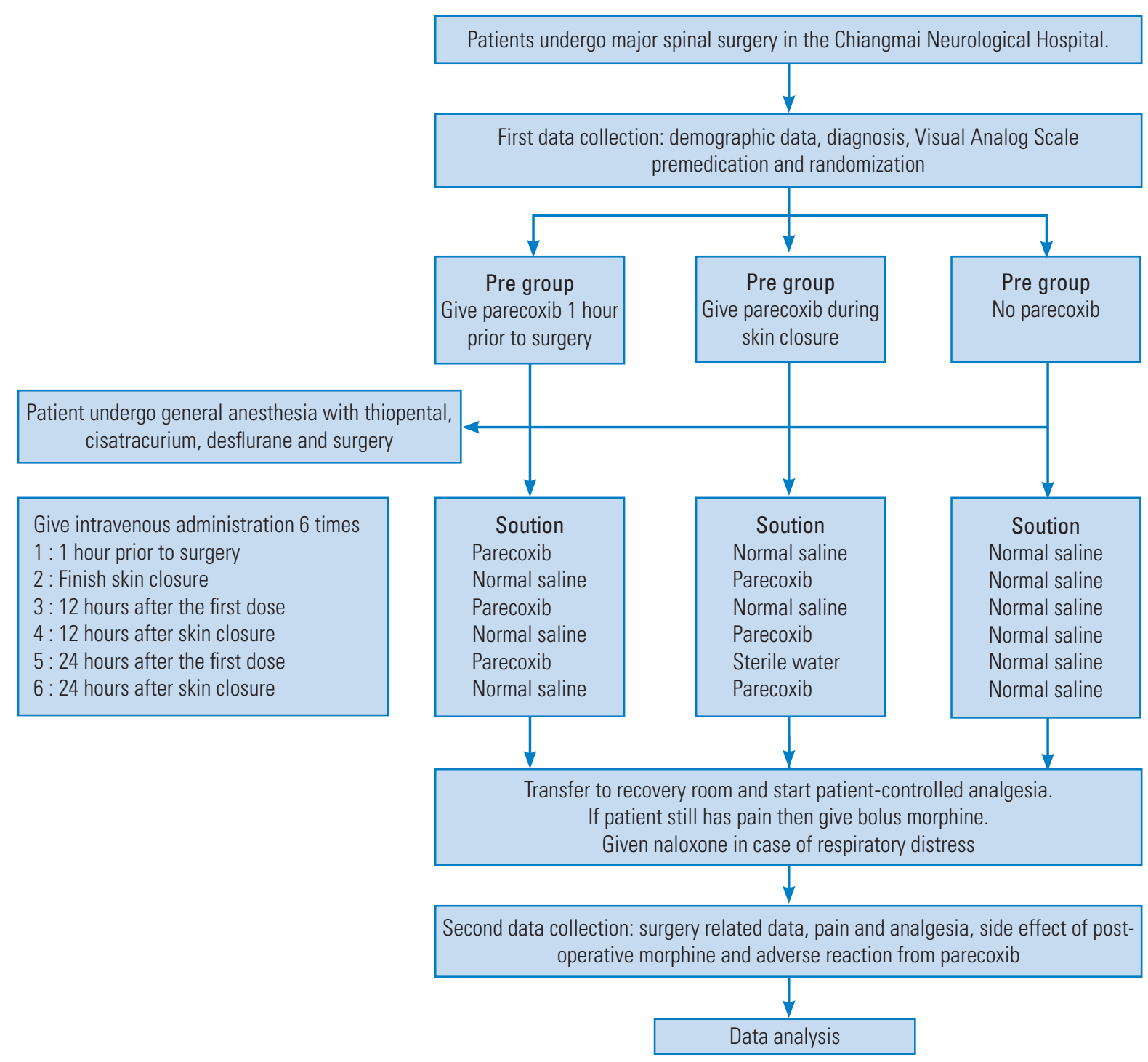

Fig. 1. Study protocol.

cal status class I-III. The exclusion criteria were as follows: (1) body weight $<50 \mathrm{~kg}$; (2) receiving any analgesic drug within 24 hours prior to surgery; (3) liver impairment; (4) renal impairment or signs of fluid retention; (5) taking fluconazole within 1 week prior to surgery; (6) hypersensitivity to parecoxib, sulfonamides, or other NSAIDs, including compounds contained in parecoxib; (7) contraindication to parecoxib administration; and (8) contraindication to lorazepam use, anesthesia medications, and morphine.

After obtaining informed consent, the researcher collected demographic data, such as age, sex, weight, height, Visual Analog Scale (VAS) scores, and data regarding diagnosis leading to surgery. The night prior to the day of surgery, all patients were premedicated with lorazepam (1 $\mathrm{mg}$ for patients $<60$ years old and $0.5 \mathrm{mg}$ for patients $>60$ years old).

On the day of surgery, the researcher used a block randomization sampling method and then divided patients into three groups: (1) pre-group, which received $40 \mathrm{mg}$ parecoxib before skin incision and at 12 and 24 hours after the first dose; (2) post-group, which received the same dose at wound closure and at 12 and 24 hours after the first dose; and (3) control group, which did not receive any parecoxib. This study was a double-blind trial. Each group received six doses of intravenous solution (Fig. 1). All solutions were colorless, and each solution was given in a $2 \mathrm{~mL}$ volume, prepared by a nurse anesthetist who was not involved in the assessment and patient care before, during, and after operation. Each solution contained 
$40 \mathrm{mg}$ parecoxib in $0.9 \%$ normal saline solution, whereas the control contained $0.9 \%$ normal saline solution only. All included participants were blinded to the intervention.

All major spine surgeries were performed by an experienced surgeon under standardized anesthesia protocol combining thiopental, cisatracurium, and desflurane in $40 \%$ oxygen and $0.1 \mathrm{mg} / \mathrm{kg}$ morphine for intraoperative pain control. Additional morphine (0.03-0.05 mg) was administered to patients when there were signs of pain, $20 \%$ higher heart rate, or blood pressure higher than the baseline. Intraoperative monitoring was performed as usual and comprised pulse oximetry, noninvasive blood pressure, electrocardiography, and capnography.

After surgery, surgical and anesthetic data, including type, location, number of spinal levels involved, duration, morphine dosage administration, and other pain relief methods, were collected. The VAS score and the use of patient-controlled analgesia (PCA) were recorded. For PCA settings, $1 \mathrm{mg} / \mathrm{mL}$ morphine was administered as an analgesic drug, with a PCA dose of $1 \mathrm{mg}$, a lockout interval of 10 minutes, and a 4-hour limit of $16 \mathrm{mg}$. Neither loading dose nor continuous drip was given. Analgesic data, including the time when the patient first used morphine from the PCA, were recorded. The VAS score was evaluated every 2 hours for the first 8 hours after surgery and then every 4 hours for the next 16 hours thereafter. Rescue medication or extra morphine was made available in cases wherein the VAS score was $\geq 5$. If the patient still experienced significant pain despite having reached the PCA 4-hour limit setting of $16 \mathrm{mg}$ morphine, a bolus of 2 mg morphine every 1 hour was given, as needed. The total morphine consumption in the first 24 hours after the operation was recorded. The ward staff would notify the researcher in case any analgesic drugs other than parecoxib and morphine were prescribed. Any patient who developed intolerable side effects from morphine was treated symptomatically. Each patient's data were collected and collated. Further, the side effects of parecoxib, such as nausea, vomiting, abdominal pain, flatulence, limb edema, dizziness, drowsiness, and oliguria, were evaluated and recorded.

Data from all three groups were recorded and analyzed. Demographic data, surgical-related data, postoperative pain, and analgesia usage were analyzed using analysis of variance and post hoc analysis with Fisher's least significant difference. Data were expressed as numbers, percentages, mean, and standard deviation. Side effects of medications after surgery were presented as numbers and percentages. All $p<0.05$ were considered statistically significant.

\section{Results}

Because of incomplete data, 17 of 144 patients were ex-

Table 1. Demographic data

\begin{tabular}{|c|c|c|c|}
\hline Characteristic & Control group ${ }^{\text {a) }}$ & Pre group ${ }^{b)}$ & Post group ${ }^{c)}$ \\
\hline Age (yr) & $60 \pm 10$ & $58 \pm 9$ & $57 \pm 9$ \\
\hline Sex (male/all) & $22 / 43$ & $15 / 42$ & $15 / 42$ \\
\hline Weight (kg) & $60.2 \pm 12.1$ & $59.1 \pm 7$ & $60.5 \pm 9.9$ \\
\hline Height $(\mathrm{cm})$ & $160 \pm 7.3$ & $157 \pm 7.2$ & $158 \pm 7.4$ \\
\hline \multicolumn{4}{|l|}{ ASA physical status } \\
\hline ASA physical status 1 & 6 & 8 & 8 \\
\hline ASA physical status 2 & 23 & 27 & 28 \\
\hline ASA physical status 3 & 14 & 7 & 6 \\
\hline Visual Analog Scale before surgery & $3.1 \pm 1.9$ & $3.2 \pm 1.7$ & $3.1 \pm 1.6$ \\
\hline Operation time (min) & $237 \pm 132$ & $244 \pm 131$ & $256 \pm 129$ \\
\hline Operated spinal level & $2 \pm 1$ & $3 \pm 1$ & $3 \pm 1$ \\
\hline $1 \%$ xylocaine with adrenaline dosage given before open skin $(\mathrm{mL})$ & $5.4 \pm 4.5$ & $6.2 \pm 4.1$ & $5.4 \pm 4.3$ \\
\hline
\end{tabular}

Values are presented as mean \pm standard deviation or number.

ASA, American Society for Anesthesiologists.

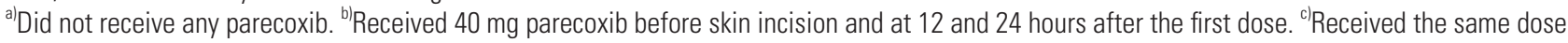
at wound closure and at 12 and 24 hours after the first dose. 
cluded from the study; nine patients withdrew consent, five had surgical complications that required intervention, and three required prolonged postoperative mechanical ventilation. Thus, data from the remaining 127 patients were analyzed. Patients were divided into three groups: 42 in the pre-group, 42 in the post-group, and 43 in the control group. Patient characteristics, type of surgery, and anesthesia doses are presented in Table 1.

Postoperative pain and analgesic data were analyzed. No significant difference was noted in the VAS scores between

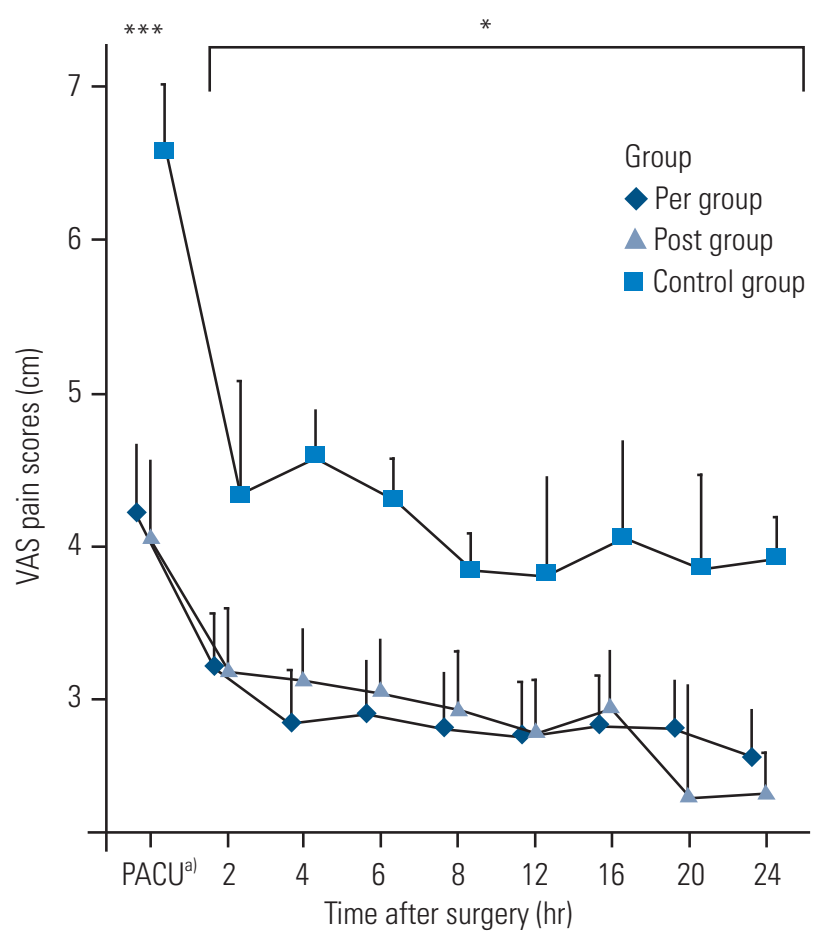

Fig. 2. VAS scores at 24 hours after surgery. Values are presented as mean \pm standard error of mean. Pre group: received $40 \mathrm{mg}$ parecoxib before skin incision and at 12 and 24 hours after the first dose; post group: received the same dose at wound closure and at 12 and 24 hours after the first dose; and control group: did not receive any parecoxib. VAS, Visual Analog Scale. ${ }^{* * *} p<0.001 ;{ }^{*} p<0.05$ vs. control group. ${ }^{\text {a) }}$ ost anesthesia care unit. the pre- and the post-groups initially, in the recovery room, and within 24 hours after surgery. However, a significant decrease was observed in the VAS score in both groups when compared with the control group (Fig. 2).

The results of pain management during and after surgery are presented in Table 2. Initial VAS score in the recovery room showed no significant difference between the pre- and post-groups; however, it was significantly less than that of the control group. The pre-group was the only group receiving parecoxib prior to surgery; however, no significant difference was noted in the total morphine consumption during surgery among all three groups and in the time taken for initial administration of morphine. However, when analyzing data for total morphine consumption at 24 hours after surgery, morphine consumption was significantly decreased in the pre-group (50\%) and also the post-group (46\%) compared with the control group. The data for total morphine consumption in 24 hours are shown in Fig. 3.

Side effects associated with postoperative analgesia included nausea and vomiting (23\%-26\%), which showed no significant differences among the three groups. No other side effects from postoperative analgesia were observed in this study, except for one patient with urinary retention in the post-group. Side effects associated with postoperative analgesia are presented in Table 3 . The most common side effects of parecoxib administration were nausea and vomiting; however, in this study, we could not distinguish whether these side effects were caused by parecoxib or morphine. Otherwise, no other side effects were reported by the patients.

\section{Discussion}

Over the years, the use of preemptive analgesia has considerably reduced, probably because several studies failed

Table 2. Analgesia during operation and postoperation

\begin{tabular}{lccc} 
Variable & Control group & Pre group & Post group $^{\mathrm{c})}$ \\
Visual Analog Scale on entering recovery room & $6.6 \pm 2.3$ & $4.2 \pm 2.9^{* * *}$ & $4.0 \pm 3.3^{* * *}$ \\
\hline Total morphine during surgery $(\mathrm{mg})$ & $8.4 \pm 2$ & $8.4 \pm 2$ & $8.9 \pm 1.8$ \\
\hline Timing for first analgesia $(\mathrm{min})$ & $71 \pm 159$ & $109 \pm 232$ & $67 \pm 115$ \\
\hline Total morphine within 24 hours after surgery $(\mathrm{mg})$ & $10.9 \pm 7.5$ & $5.4 \pm 4.7^{* * *}$ & $5.9 \pm 5.5^{* * *}$ \\
\hline
\end{tabular}

Values are presented as mean \pm standard deviation.

${ }^{* * *} p<0.001$ (vs. control group). ${ }^{\text {al }}$ Did not receive any parecoxib. ${ }^{\text {b) Received }} 40 \mathrm{mg}$ parecoxib before skin incision and at 12 and 24 hours after the first dose. ${ }^{c}$ Received the same dose at wound closure and at 12 and 24 hours after the first dose. 
to show any substantial advantage of preoperative analgesia over postoperative analgesia [16,17]. Several randomized, double-blind, placebo-controlled trials demonstrated the beneficial effects of parecoxib after surgery, including decrease in pain intensity and postoperative opioid consumption [18-24]. However, a number of studies investigated the parecoxib analgesic effect both during and after surgery. The results were still inconclusive because some studies showed a benefit in decreasing both the pain score and opioid consumption $[25,26]$, but some studies showed no difference $[27,28]$. Hence, several comparative studies were conducted to investigate the difference of the parecoxib analgesic effect between the pre- and post-

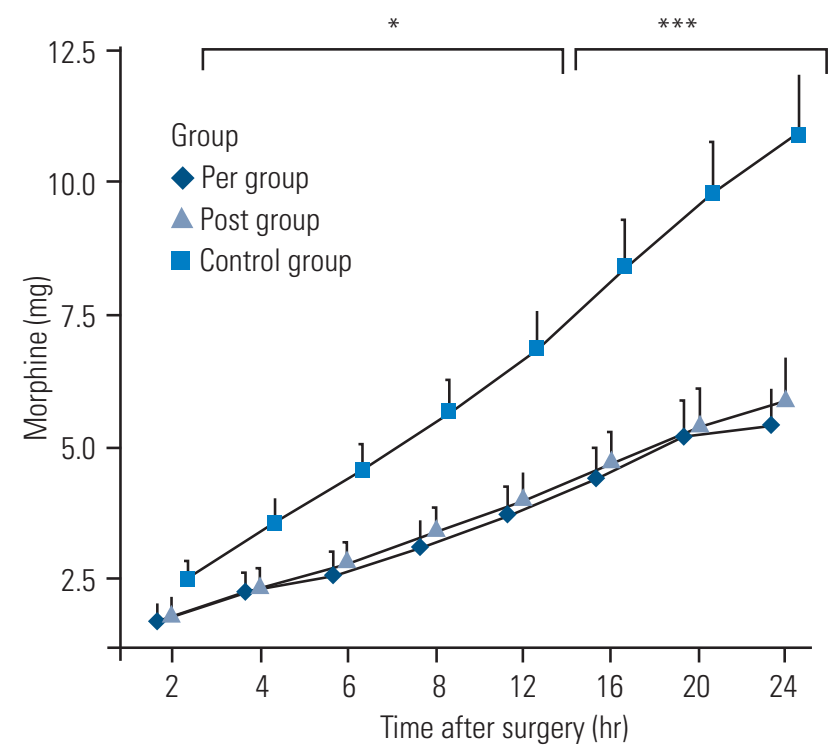

Fig. 3. Morphine dosage in the first 24 hours after surgery. Values are presented as mean \pm standard error of mean. Pre group: received $40 \mathrm{mg}$ parecoxib before skin incision and at 12 and 24 hours after the first dose; post group: received the same dose at wound closure and at 12 and 24 hours after the first dose; and control group: did not receive any parecoxib. ${ }^{* * *} p<0.001 ;{ }^{*} p<0.05$ vs. control group. operative period. Riest et al. [12] compared patients who underwent a discectomy. They found that parecoxib must be administered both pre- and postoperatively to decrease the pain score and side effects of opioid. A single-dose of parecoxib administered either preoperatively or postoperatively did not reduce both the pain score and side effects of morphine. Also, in the study conducted by Martinez et al. [13], both patient groups that received parecoxib preand post-surgery had significantly reduced postoperative morphine consumption when compared with placebo. However, the VAS score was significantly reduced only between 12 and 24 hours after surgery. Pandazi et al. [14] and Bao et al. [15] investigated postoperative pain control in colorectal and total hip arthroplasty, respectively, comparing preincisional versus postincisional parecoxib administration (30 minutes before and after incision). The study conducted by Pandazi et al. [14] reported that preincisional and postincisional administration of parecoxib did not make a substantial difference in terms of the postoperative pain score and incidence of morphine-related side effects. However, preincisional parecoxib administration resulted in significant reduction of morphine usage after surgery when compared with postincisional administration. In contrast, the results of the study conducted by Bao et al. [15] were quite different, showing that preincisional parecoxib administration significantly reduced postoperative pain and the total amount of morphine consumption.

Previous studies, as mentioned above, did compare preoperative and postoperative parecoxib administration, which showed inconclusive results because the most effective timing for parecoxib administration was not considered. Theoretically, preoperative parecoxib administration can reduce the peripheral modulator of nociceptors and inflammation, which could lead to reduction of postoperative pain and the amount of morphine required. In

Table 3. Prevalence of side effect from morphine

\begin{tabular}{lccc} 
Variable & Control group $\left.{ }^{\mathrm{a}}\right)$ & Pre group $^{\mathrm{b})}$ & Post group $^{c)}$ \\
Postoperative nausea and vomiting & $11(26.2)$ & $10(23.8)$ & $11(25.6)$ \\
Respiratory depression & 0 & 0 & 0 \\
Drowsiness & 0 & 0 & 0 \\
Urinary retention & 0 & 0 & $1(2.3)$ \\
\hline
\end{tabular}

Values are presented as number (\%).

${ }^{a}$ Did not receive any parecoxib. ${ }^{b}$ Received $40 \mathrm{mg}$ parecoxib before skin incision and at 12 and 24 hours after the first dose. ${ }^{c / R e c e i v e d ~ t h e ~ s a m e ~ d o s e ~}$ at wound closure and at 12 and 24 hours after the first dose. 
fact, there were other associated factors, such as operative time, which may have affected the effectiveness of analgesia administration between the pre- and postoperative period. Riest et al. [12] studied discectomy surgery but similarly did not consider the duration of operative times or the most effective timing of pre- and postoperative parecoxib administration. Martinez et al. [13] studied surgical procedures that had variable operation times $(118 \pm 35$ minutes); hence, it is possible that the effective duration of parecoxib was not enough to cover the entire operation when the surgery was prolonged. Pandazi et al. [14] and Bao et al. [15] set the length of the duration for parecoxib administration (pre- and post-incision) at approximately 1 hour (30 minutes before and after incision). As evident from these studies, the difference in the length of duration times between pre- and postoperative parecoxib administration may affect the results.

This study involved patients who underwent major spinal surgeries with operation times $>2$ hours. The pregroup's first dose of parecoxib was administered at 1 hour prior to the start of the operation, whereas the postgroup's first dose was administered at the time of skin closure. Parecoxib was not administered during the operation in either group. The reason for separating administration times as much as possible was to increase the difference in the parecoxib analgesic level. In the pre-group, which received parecoxib at 1 hour prior to surgery, it is possible that after the operation, these patients could experience more pain upon waking up, which requires more morphine from the PCA than the post-group that received parecoxib at the time of skin closure. This might be due to the lower analgesic level of parecoxib at the time the operation was finished. Also, in this study, parecoxib was continued every 12 hours as preventive analgesia after the first parecoxib dose along with the PCA morphine and rescue medication to further evaluate the efficacy of the medication administered at different times.

This study showed no significant difference in the VAS score, amount of morphine consumption (during and post-surgery), or side effects from morphine in both the pre- and post-groups. These groups significantly differed in terms of the administration of parecoxib compared with the control group, which showed a reduction in the VAS score and morphine consumption. Thus, it is implied that this study supported neither preemptive analgesia nor the timing of parecoxib administration, but showed obvious benefits when compared with the group that did not receive parecoxib (control group). In the study conducted by Martinez et al. [13], parecoxib was administered both pre- and postoperatively, as in this study. At the end of the operation, the parecoxib level in the blood was low. The main difference between the previous study and this study is the operation time. Martinez et al. [13] studied total hip replacements, which had shorter operation times, i.e., at least 2 hours, compared with the present study on major spine surgery; however, in this study, preoperative parecoxib was administered at 1 hour prior to surgery, which made the time interval between pre- and postoperative parecoxib administration at least 3 hours. However, the results of both studies were quite similar at several times owing to the adequate use of continuous preventive analgesia after surgery.

This study has several limitations. First, the side effects of parecoxib were not completely determined, because the most common side effects, such as nausea and vomiting, could not be distinguished from those of morphine. Information on other side effects determined through patient questionnaires and physical examinations were also collected, but laboratory examinations, such as blood urea nitrogen, creatinine, lactate dehydrogenase, creatinine phosphokinase, blood sugar, etc., were not performed. Second, the VAS score is considered a subjective data with individual differences. Third, spine disease has a neuropathic component of pain. Spine surgery can cause nerve injury leading to postoperative neuropathic pain, which was not examined in this study. Moreover, parecoxib has is less efficient in neuropathic pain treatment. Because this study failed to obtain laboratory data or include neuropathic pain data, future studies should be conducted to include these.

The results of this study can be possibly applied to major spinal surgery. Because the timing of parecoxib administration does not affect postoperative analgesia, it is suggested that the most suitable time for administration of parecoxib should be during the skin closure period, with continued medication every 12 hours along with PCA morphine for rescue medication to maximize analgesic efficacy. Moreover, the delayed initial dose may reduce the total dose of parecoxib usage. Further studies are needed to investigate the cost-effectiveness of pre- and postoperative parecoxib administration. 


\section{Conclusions}

The timing of parecoxib administration, i.e., either before or after surgery, does not affect the analgesic efficacy, as evaluated by the VAS score and morphine consumption within the first 24 hours after surgery. It also does not affect the safety of pain management with parecoxib.

\section{Conflict of Interest}

No potential conflict of interest relevant to this article was reported.

\section{Acknowledgments}

On behalf of the investigators, the authors gratefully acknowledge the support from Associate Professor Yodying Punjasawadwong, Department of Anesthesiology, Faculty of Medicine, Chiangmai University, Chiangmai, and Dr. Phuping Akavipat, Department of Anesthesiology, Prasat Neurological Institute, Bangkok, Thailand. Funding for this study was provided by Department of Medical Service fund, Ministry of Public Health, Thailand. The investigators hope that this research will be beneficial to healthcare providers in acute postoperative pain management.

\section{References}

1. Pumberger M, Chiu YL, Ma Y, Girardi FP, Mazumdar M, Memtsoudis SG. National in-hospital morbidity and mortality trends after lumbar fusion surgery between 1998 and 2008. J Bone Joint Surg Br 2012;94:359-64.

2. Rajaee SS, Bae HW, Kanim LE, Delamarter RB. Spinal fusion in the United States: analysis of trends from 1998 to 2008. Spine (Phila Pa 1976) 2012;37:6776.

3. Buvanendran A, Thillainathan V. Preoperative and postoperative anesthetic and analgesic techniques for minimally invasive surgery of the spine. Spine (Phila Pa 1976) 2010;35(26 Suppl):S274-80.

4. Wu CL, Raja SN. Treatment of acute postoperative pain. Lancet 2011;377:2215-25.

5. Mathiesen O, Dahl B, Thomsen BA, et al. A comprehensive multimodal pain treatment reduces opioid consumption after multilevel spine surgery. Eur Spine J 2013;22:2089-96.
6. Garcia RM, Cassinelli EH, Messerschmitt PJ, Furey CG, Bohlman HH. A multimodal approach for postoperative pain management after lumbar decompression surgery: a prospective, randomized study. J Spinal Disord Tech 2013;26:291-7.

7. Gottschalk A, Freitag M, Tank S, et al. Quality of postoperative pain using an intraoperatively placed epidural catheter after major lumbar spinal surgery. Anesthesiology 2004;101:175-80.

8. Gottschalk A, Durieux ME, Nemergut EC. Intraoperative methadone improves postoperative pain control in patients undergoing complex spine surgery. Anesth Analg 2011;112:218-23.

9. Gianesello L, Pavoni V, Barboni E, Galeotti I, Nella A. Perioperative pregabalin for postoperative pain control and quality of life after major spinal surgery. J Neurosurg Anesthesiol 2012;24:121-6.

10. Alanoglu Z, Ates Y, Orbey BC, Turkcapar AG. Preoperative use of selective COX-II inhibitors for pain management in laparoscopic nissen fundoplication. Surg Endosc 2005;19:1182-7.

11. Pfizer Medical Information. Dynastat (Parecoxib sodium) prescribing information [Internet]. West Ryde (NSW): Pfizer; c2016 [cited 2015 Oct 20]. Available from: http://www.pfizer.com.au/products/dynastat.

12. Riest G, Peters J, Weiss M, et al. Preventive effects of perioperative parecoxib on post-discectomy pain. $\mathrm{Br}$ J Anaesth 2008;100:256-62.

13. Martinez V, Belbachir A, Jaber A, et al. The influence of timing of administration on the analgesic efficacy of parecoxib in orthopedic surgery. Anesth Analg 2007;104:1521-7.

14. Pandazi A, Kapota E, Matsota P, Paraskevopoulou P, Dervenis C, Kostopanagiotou G. Preincisional versus postincisional administration of parecoxib in colorectal surgery: effect on postoperative pain control and cytokine response: a randomized clinical trial. World J Surg 2010;34:2463-9.

15. Bao Y, Fang J, Peng L, et al. Comparison of preincisional and postincisional parecoxib administration on postoperative pain control and cytokine response after total hip replacement. J Int Med Res 2012;40:1804-11.

16. Brennan TJ, Taylor BK. Analgesic treatment before incision compared with treatment after incision provides no improvement in postoperative pain relief. J Pain 2000;1:96-8. 
17. Moiniche S, Kehlet H, Dahl JB. A qualitative and quantitative systematic review of preemptive analgesia for postoperative pain relief: the role of timing of analgesia. Anesthesiology 2002;96:725-41.

18. Viscusi ER, Gimbel JS, Halder AM, Snabes M, Brown MT, Verburg KM. A multiple-day regimen of parecoxib sodium $20 \mathrm{mg}$ twice daily provides pain relief after total hip arthroplasty. Anesth Analg 2008;107:652-60.

19. Malan TP Jr, Marsh G, Hakki SI, Grossman E, Traylor L, Hubbard RC. Parecoxib sodium, a parenteral cyclooxygenase 2 selective inhibitor, improves morphine analgesia and is opioid-sparing following total hip arthroplasty. Anesthesiology 2003;98:950-6.

20. Apfelbaum JL, Desjardins PJ, Brown MT, Verburg KM. Multiple-day efficacy of parecoxib sodium treatment in postoperative bunionectomy pain. Clin J Pain 2008;24:784-92.

21. Zhu Y, Wang S, Wu H, Wu Y. Effect of perioperative parecoxib on postoperative pain and local inflammation factors PGE2 and IL-6 for total knee arthroplasty: a randomized, double-blind, placebo-controlled study. Eur J Orthop Surg Traumatol 2014;24:395-401.

22. Gehling M, Arndt C, Eberhart LH, Koch T, Kruger T, Wulf H. Postoperative analgesia with parecoxib, acetaminophen, and the combination of both: a randomized, double-blind, placebo-controlled trial in patients undergoing thyroid surgery. Br J Anaesth 2010;104:761-7.
23. Tang J, Li S, White PF, Chen X, et al. Effect of parecoxib, a novel intravenous cyclooxygenase type2 inhibitor, on the postoperative opioid requirement and quality of pain control. Anesthesiology 2002;96:1305-9.

24. Barton SF, Langeland FF, Snabes MC, et al. Efficacy and safety of intravenous parecoxib sodium in relieving acute postoperative pain following gynecologic laparotomy surgery. Anesthesiology 2002;97:306-14.

25. Jirarattanaphochai K, Thienthong S, Sriraj W, et al. Effect of parecoxib on postoperative pain after lumbar spine surgery: a bicenter, randomized, doubleblinded, placebo-controlled trial. Spine (Phila Pa 1976) 2008;33:132-9.

26. Desjardins PJ, Grossman EH, Kuss ME, et al. The injectable cyclooxygenase-2-specific inhibitor parecox$\mathrm{ib}$ sodium has analgesic efficacy when administered preoperatively. Anesth Analg 2001;93:721-7.

27. Akaraviputh T, Leelouhapong C, Lohsiriwat V, Aroonpruksakul S. Efficacy of perioperative parecox$\mathrm{ib}$ injection on postoperative pain relief after laparoscopic cholecystectomy: a prospective, randomized study. World J Gastroenterol 2009;15:2005-8.

28. Luscombe KS, McDonnell NJ, Muchatuta NA, Paech MJ, Nathan EA. A randomized comparison of parecoxib versus placebo for pain management following minor day stay gynaecological surgery. Anaesth Intensive Care 2010;38:141-8. 